\title{
Elevated Serum Transferrin Saturation and Mortality
}

\author{
Arch G. Mainous III, $P b D^{1}$ \\ James M. Gill, MD, MPH \\ Peter J. Carek, $M D, M S^{2}$ \\ 'Department of Family Medicine, Medical \\ University of South Carolina, Charleston, SC \\ ${ }^{2}$ Department of Family \& Community \\ Medicine, Christiana Care Health System, \\ Wilmington, Del
}

\begin{abstract}
BACKGROUND A large proportion of US adults have elevated transferrin saturation, an indicator of a predisposition for iron overload. The purpose of this study was to evaluate the relationship between elevated serum transferrin saturation and mortality.

METHODS This cohort study was conducted using data from the First Health and Nutrition Examination Survey I (1971-1974) (NHANES I) merged with the NHANES I Epidemiologic Followup Study (1992) ( $N=10,714)$. We used SUDAAN and appropriate weights to make population estimates for the adult US population (aged 25 to 74 years at baseline). All-cause mortality was evaluated in relation to serum transferrin saturation of greater than $45 \%$, greater than $50 \%$, greater than $55 \%$, and greater than $60 \%$ using Cox proportional hazards regression.
\end{abstract}

RESULTS In a Cox proportional hazards model controlling for potential confounders, including comorbid diseases, smoking, and cholesterol, all-cause mortality is significantly greater for persons with a serum transferrin saturation of more than $55 \%$, compared with those with saturations below this cutoff (hazards ratio $[\mathrm{HR}]=1.60,95 \%$ confidence interval $[\mathrm{Cl}], 1.17-2.21)$. No one who died had hemochromatosis as any of the 20 listed causes of death. Many of the underlying causes of death for persons with serum transferrin saturation levels of more than $55 \%$ are common causes of death in the general population, although these persons were more likely to have died of cirrhosis and diabetes, a finding consistent with iron overload.

CONCLUSIONS In this nationally representative cohort of adults, those with elevated serum transferrin saturation, more than $2 \%$ of the adult US population, were at increased risk for all-cause mortality.

Ann Fam Med 2004;2:133-138. DOI: 10.1370/afm.25.

\section{INTRODUCTION}

$\mathrm{E}$ xcess iron and iron overload can facilitate the occurrence of a variety of diseases, including cirrhosis, congestive heart failure, diabetes, and cancer. ${ }^{1-4}$ Pathologic iron accumulation affects many organs, such as the liver, pancreas, and heart, among others. ${ }^{5}$ Hereditary iron overload is characterized by excessive intestinal absorption and cellular deposition of iron and is found in several conditions, including hemochromatosis, African iron overload, and iron-loading anemias. ${ }^{2,4,6}$ Hemochromatosis is among the most common inherited metabolic abnormalities, particularly among those of Northern European origin. ${ }^{7-11}$

Serum transferrin saturation is a commonly used indicator for a predisposition of iron overload, and its measurement is a frequent first step in screening protocols for hereditary hemochromatosis. ${ }^{5}$ A substantial proportion of adults in the United States have elevated transferrin saturation levels, although few have a diagnosed iron overload condition. ${ }^{9}$ Whereas iron overload facilitates certain disease processes, it is unclear whether elevated transferrin saturation is a risk factor for mortality. 
Although several studies have focused on the mortality risk associated with hemochromatosis, methodological limitations and population definitions suggest additional investigation into the risk associated with transferrin saturation is warranted. Several cross-sectional studies have attempted to examine the mortality risk of being homozygous for the C282Y mutation of the HFE gene by investigating whether homozygotes were underrepresented in older age-groups. ${ }^{12,13}$ These studies found that homozygotes were not underrepresented and argued that life-threatening disease might not occur in many C282Y homozygotes. These studies, however, were not cohort studies assessing survival time or mortality risk, and they defined their population based on a genetic marker rather than a clinical indicator (eg, transferring saturation). Other studies have been based on patients with a diagnosis of hemochromatosis. ${ }^{14,15}$ These additional studies are based on patients with advanced disease as evidenced by baseline rates of $68 \%$ for cirrhosis. Because few patients with elevated serum transferrin saturation levels ever have hemochromatosis diagnosed, estimating risk based on this advanced disease population might not indicate the true risk of iron overload or elevated transferrin saturation. ${ }^{16}$

It is unclear whether elevated serum transferrin saturation has an unrecognized deleterious affect on survival. ${ }^{16,17}$ The purpose of this study, therefore, was to examine the mortality risk among a nationally representative cohort of persons who have elevated serum transferrin saturation levels.

\section{METHODS}

The design of this study is a retrospective cohort study of persons aged 25 to 74 years at the time of the index interview. The data sources used in this study are the First Health and Nutrition Examination Survey I (1971-1974) (NHANES I) merged with the NHANES I Epidemiologic Followup Study (1992) (NHEFS).

NHANES I was multistage, stratified probability survey designed to collect extensive demographic, medical history, nutritional, clinical, and laboratory data representative of the noninstitutionalized civilian US population.

The NHANES I Epidemiologic Followup Study is a national longitudinal study designed to investigate the relationships between clinical, nutritional, and behavioral factors assessed at baseline NHANES I and subsequent morbidity, mortality, and institutionalization. The NHEFS initial population includes the 14,407 participants who were 25 to 74 years of age when first examined in NHANES I. More than $98 \%$ of those in the initial NHANES I cohort were traced and supplied data in the NHEFS.
The follow-up information was gathered in 1 of 3 ways. Subjects were interviewed who could be contacted and could participate. Surviving subjects were always administered the subject questionnaire. If the subject was alive but incapacitated, a slightly modified version of the subject questionnaire was administered to a proxy respondent. A separate proxy questionnaire was used only when the person had died. Finally, for those who had died during the period between the NHANES I index interview and the follow-up interview, information from a death certificate was recorded.

\section{Transferrin Saturation}

In the original NHANES I, serum transferrin saturation was measured. We defined elevated serum transferrin saturation as greater than $45 \%$, greater than $50 \%$, greater than $55 \%$, and greater than $60 \%$. All of these cutoff values had previously been proposed or used in population-based studies of elevated serum transferrin saturation. ${ }^{18,19}$ The serum transferrin saturation percentage was calculated by dividing the serum iron level by total iron-binding capacity.

\section{Mortality}

Mortality was measured as all-cause mortality. It has been contended that all-cause mortality is an endpoint more unbiased than disease-specific mortality. ${ }^{20}$ The mortality variable was assessed using death certificates. The cause of death was measured as the underlying cause of death.

\section{Control Variables}

We attempted to examine the independent relationship between elevated serum transferrin saturation and mortality while controlling for potential confounders. Control variables that were available in the NHANES I baseline data set were age, sex, race, poverty status, and education. Insurance was not available in this data set, but poverty status, which is an index based on income and number of household members, was. Comorbidities were controlled for at baseline by positive responses to questions regarding whether a physician ever told the patient that he or she had any of 42 different conditions (eg, diabetes, high blood pressure, hepatitis). The Charlson Comorbidity Index was calculated from the responses to these questions. ${ }^{21}$ We also included total serum cholesterol and smoking status at baseline as control variables for mortality risk.

\section{Data Analysis}

For the analysis of the NHEFS we used sampling weights to calculate prevalence estimates for the civilian noninstitutionalized US population aged 25 to 74 years in 1971 to 1974 . Because of the complex sampling 
Table 1. Characteristics of the Population Collected in NHANES I Baseline (aged 25 to 74 years)

\begin{tabular}{|c|c|c|}
\hline Characteristic & $\begin{array}{c}\text { Percent of Total } \\
(\mathrm{N}=104,124,97)\end{array}$ & $\begin{array}{c}\text { Percent with } \\
\text { Transferrin } \\
\text { Saturation }>55 \% \\
(\mathrm{~N}=2,868,869)\end{array}$ \\
\hline \multicolumn{3}{|l|}{ Age, years } \\
\hline $25-34$ & 25.6 & 24.6 \\
\hline $35-52$ & 39.2 & 42.1 \\
\hline $53-69$ & 30.1 & 28.0 \\
\hline $70+$ & 5.1 & 5.3 \\
\hline \multicolumn{3}{|l|}{ Sex } \\
\hline Male & 47.3 & 54.7 \\
\hline Female & 52.7 & 45.3 \\
\hline \multicolumn{3}{|l|}{ Race* } \\
\hline White & 89.3 & 93.8 \\
\hline Negro & 9.8 & 5.3 \\
\hline Other & 0.9 & 0.9 \\
\hline \multicolumn{3}{|l|}{ Poverty Index ${ }^{\dagger}$} \\
\hline At or below & 15.1 & 18.2 \\
\hline Above & 84.9 & 81.8 \\
\hline \multicolumn{3}{|l|}{ Education } \\
\hline None & 0.9 & 0.8 \\
\hline Primary school & 19.8 & 14.8 \\
\hline High school & 52.2 & 46.1 \\
\hline College & 20.5 & 23.9 \\
\hline College graduate & 6.7 & 14.3 \\
\hline \multicolumn{3}{|c|}{ Note: Population estimates for the US population aged 25 to 74 years in 1971.} \\
\hline \multicolumn{3}{|c|}{ NHANES I = First National Health and Nutrition Examination Survey. } \\
\hline \multicolumn{3}{|c|}{$\begin{array}{l}\text { * Terms used for classification by the NHANES I in } 1971 . \\
\text { † As calculated in } 1971 .\end{array}$} \\
\hline
\end{tabular}

design of the survey, we performed all analyses with SUDAAN. ${ }^{22}$ Our cohort for analysis was limited to those with data in both the baseline and follow-up data sets and who had their serum transferrin saturation measured $(\mathrm{N}=10,714)$.

We examined the relationship between elevated serum transferrin saturation and proportion of the cohort surviving throughout the follow-up period. We computed Kaplan-Meier curves for mortality and time in years since the baseline examination for elevated serum transferrin saturation at $55 \%$, because this level has been suggested as an action point for further investigation of iron overload..$^{23}$ Results provided unadjusted estimates of the all-cause mortality rate for those with and without elevated serum transferrin saturation. Finally, we performed Cox regressions with survival time for serum transferrin saturation levels in 3 different models. The first model defined the population into several categories of transferrin saturation at baseline ( $45 \%$ [reference category] or less, $45.1 \%$ to $50.0 \%$, $50.1 \%$ to $55.0 \%, 55.1 \%$ to $60.0 \%$, and greater than $60 \%$ ) controlling for age, sex, race, poverty status, education, total serum cholesterol level $(200 \mathrm{mg} / \mathrm{dL}[5.20$ $\mathrm{mmol} / \mathrm{L}$ ] or higher or less than $200 \mathrm{mg} / \mathrm{dL}$ ), current smoking status, and comorbidities. The second and third models were computed examining the population as individuals having elevated or normal transferrin saturation percentage, defining elevated transferrin saturation as greater than $55 \%$ in one model and greater than $60 \%$ in another. These models were computed for all-cause mortality. In these models, survival time was a continuous variable measured in 1-year increments.

\section{RESULTS}

Table 1 displays the characteristics of the population. The proportion of the population with elevated serum transferrin saturation was $6.8 \%$ at more than $45 \%$ saturation, $3.8 \%$ at more than $50 \%$ saturation; $2.3 \%$ at more than $55 \%$; and $1.3 \%$ at more than $60 \%$ saturation. As displayed in Table 1, those with transferrin saturation levels of greater than $55 \%$ are more likely to be male, white, and college educated, compared with the overall population.

Figure 1 shows the cumulative, unadjusted all-cause mortality with time for persons who had elevated transferrin saturations at $55 \%$. After controlling for confounding variables in a Cox proportional hazards model (Table 2), all-cause mortality risk is significantly greater for those with a transferrin saturation of $60 \%$ compared with those who had a transferrin saturation of $45 \%$ or less. When the entire population is dichotomized into elevated or not elevated transferrin saturation levels at $55 \%$, however, persons with elevated transferrin saturation levels have a significantly increased mortality risk (hazards ratio [HR] 1.60, 95\% confidence interval [CI], 1.17-2.21). Mortality risk is also significantly greater for those with a transferrin saturation of greater than $60 \%$, compared with those at less than this cutoff.

No one in the cohort who died had hemochromatosis as any of the 20 listed causes of death. Table 3 shows the top 10 causes of death for those with a transferrin saturation of more than $55 \%$. Compared with those who had a transferrin saturation of $55 \%$ or less, both groups have 5 of the 10 causes of death in common. Consistent with the descriptions of iron overload, however, cirrhosis and diabetes were more common underlying causes of death among the elevated transferrin saturation cohort.

\section{DISCUSSION}

The current study indicates that persons with a serum transferrin saturation greater than $55 \%$ are at increased risk of all-cause mortality. Elevated serum transferrin levels are associated with decreased survival, even after controlling for factors such as comorbid conditions (eg, hypertension, diabetes, cancer), smoking, and elevated choles- 
Figure 1 . All-cause mortality at $>55 \%$ transferrin saturation.

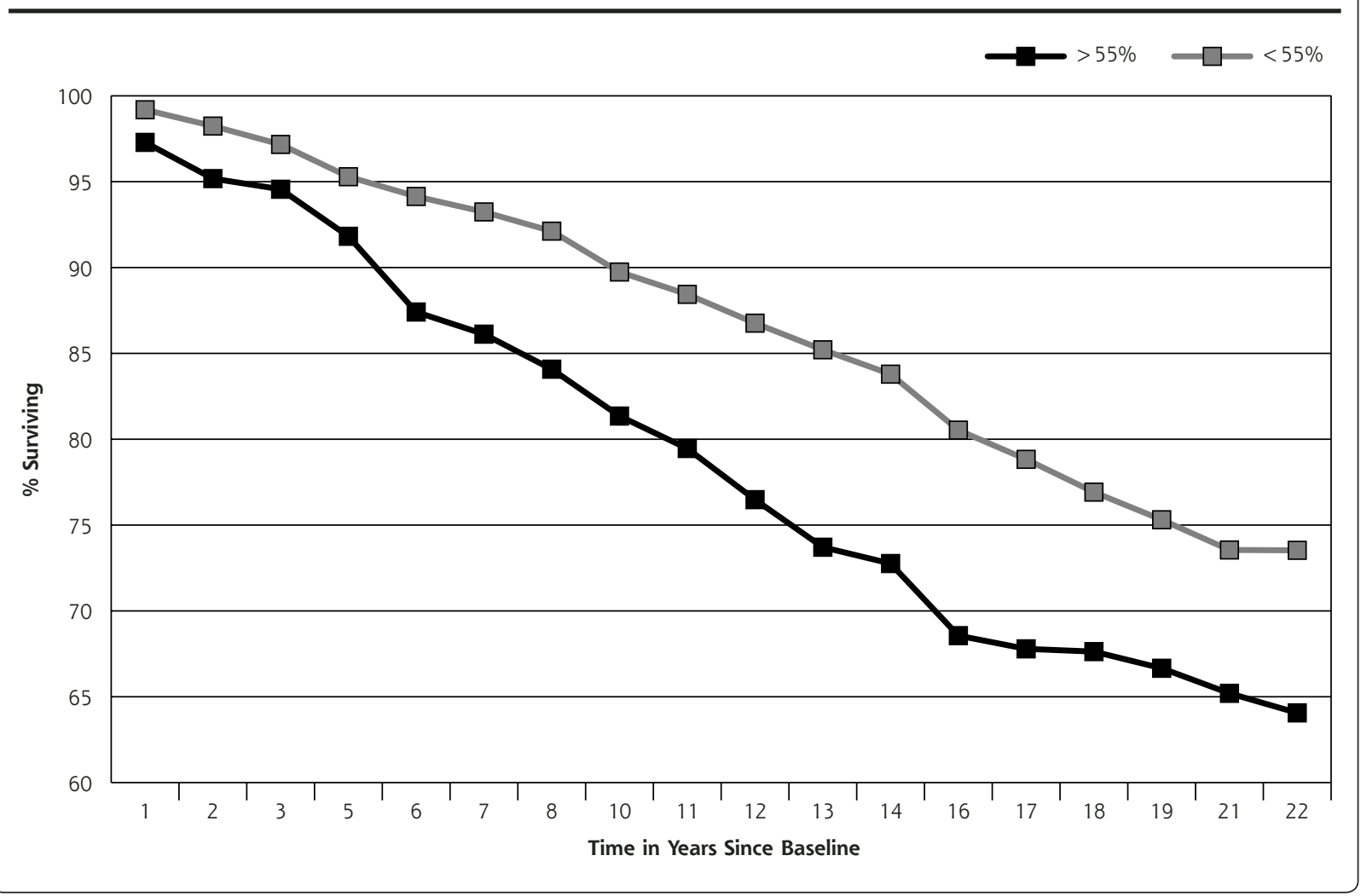

terol levels. Historically, the morbidity and mortality of iron overload diseases has been associated with cirrhosis and other liver diseases, diabetes mellitus, and cardiomyopathy. These findings provide new information on the impact of elevated serum transferrin saturation.

Approximately $2 \%$ of the adult US population has transferrin saturation levels greater than 55\%. According to estimates from the early 1990s, more than 3.3 million US adults have an elevated transferrin saturation according to this criteria. ${ }^{16}$ This risk factor for mortality is essentially unrecognized in a substantial proportion of adults. To provide some context, the prevalence of diabetes, both diagnosed and undiagnosed, is estimated at approximately $8 \%$ of the population. ${ }^{24}$

Although the current study has found an association between elevated transferrin saturation and mortality, the underlying pathophysiologic mechanism associated with the increased risk is unclear. Consistent with the pathophysiologic mechanism of hereditary hemochromatosis and African iron overload, an elevation in serum transferrin saturation might represent an iron overload state with accumulation of iron in vital organs.

The findings of this study indicating a relatively underinvestigated risk factor for mortality have similarities to those of recent investigations focusing on C-reactive protein (CRP). C-reactive protein was first associated with cardiovascular disease when it was noticed that CRP was increased after an acute myocardial infarction. ${ }^{25}$ It was unclear what this marker represented as an acute-phase reactant, even though it had a consistent relationship with cardiovascular

\section{Table 2. Adjusted Cox Regression Models of Elevated Serum Transferrin Saturation and All-Cause Mortality*}

\begin{tabular}{lcc}
\hline & Hazards Ratio & $95 \% \mathbf{C l}$ \\
\hline Model 1 & & \\
$\leq 45 \%$ (reference category) & 1.00 & --- \\
$45.1-50.0$ & 0.93 & $0.72-1.21$ \\
$50.1-55.0$ & 0.82 & $0.57-1.19$ \\
$55.1-60.0$ & 1.50 & $0.96-2.35$ \\
$>60 \%$ & 1.67 & $1.08-2.58$ \\
Model 2 & & \\
$\leq 55 \%$ (reference category) & 1.00 & --- \\
$>55 \%$ & 1.60 & $1.17-2.21$ \\
Model 3 & 1.00 & -- \\
$\leq 60 \%$ (reference category) & 1.67 & $1.08-2.58$ \\
$>60 \%$ & & \\
\hline Cl $=$ confidence interval. & & \\
${ }^{*}$ Controlling for age, race, sex, education, poverty, total serum cholesterol, smok- \\
ing, and comorbidities.
\end{tabular}




\section{Table 3. Ten Leading Underlying Causes of Death for Those with Serum Transferrin Saturation $>55 \%$ and $\leq 55 \%$}

\begin{tabular}{|c|c|}
\hline ICD Code & $\begin{array}{l}\text { Percent of } \\
\text { Total Deaths }\end{array}$ \\
\hline \multicolumn{2}{|l|}{$>55 \%$ Transferrin saturation } \\
\hline 429.2 Cardiovascular disease, unspecified & $17.6^{*}$ \\
\hline 414.0 Coronary atherosclerosis & $8.8^{*}$ \\
\hline 410 Acute myocardial infarction & $7.6^{*}$ \\
\hline 571.5 Cirrhosis of liver without mention of alcohol & 6.7 \\
\hline 250.0 Diabetes mellitus & 6.0 \\
\hline 414.9 Chronic ischemic heart disease, unspecified & $4.2^{*}$ \\
\hline 571.2 Alcoholic cirrhosis of the liver & 3.0 \\
\hline 200.1 Hodgkin's paragranuloma & 2.7 \\
\hline 188.9 Malignant neoplasm of bladder, unspecified & 2.7 \\
\hline $\begin{array}{l}\text { 162.9 Malignant neoplasm of bronchus and lung, } \\
\text { unspecified }\end{array}$ & $2.7^{*}$ \\
\hline \multicolumn{2}{|l|}{$\leq 55 \%$ Transferrin saturation } \\
\hline 410 Acute myocardial infarction & $18.7^{*}$ \\
\hline 414.0 Coronary atherosclerosis & $8.0^{*}$ \\
\hline $\begin{array}{l}\text { 162.9 Malignant neoplasm of bronchus and lung, } \\
\text { unspecified }\end{array}$ & $6.0^{*}$ \\
\hline 436 Acute cerebrovascular disease & 3.6 \\
\hline 496 Chronic airway obstruction & 3.3 \\
\hline 429.2 Cardiovascular disease, unspecified & $3.2^{*}$ \\
\hline 414.9 Chronic ischemic heart disease, unspecified & $2.8^{*}$ \\
\hline 174.9 Malignant neoplasm of female breast & 2.6 \\
\hline 486 Pneumonia & 2.2 \\
\hline 153.9 Malignant neoplasm of colon, unspecified & 1.9 \\
\hline
\end{tabular}

disease outcomes. ${ }^{26}$ Much as the findings of the present study between baseline transferrin saturation and survival risk 20 years later, CRP was a strong predictor of risk even 20 years after initial blood samples were obtained. ${ }^{27} \mathrm{C}$-reactive protein is now recommended as useful in cardiovascular disease detection and prevention. ${ }^{26}$ Further research is needed to determine whether useful prognostic information is yielded from measures of transferrin saturation.

A strength of this study is that it uses a national population estimate as a cohort. Further, the results are based on elevated serum transferrin saturation rather than on a specific diagnosis, such as hemochromatosis. Because hemochromatosis is so rarely diagnosed, population level estimates of morbidity or mortality using persons with hemochromatosis might represent implications only for those with advanced disease manifesting a classic presentation.

Several limitations to these results should be noted. First, the estimate from the NHANES I was based on elevated serum transferrin saturation measurements, an appropriate first step in determining a predisposition to iron overload. Serum transferrin saturation can be considered a phenotypic marker of homozygosity for hemochromatosis but by itself does not indicate the presence or absence of iron overload. Thus, a single elevated transferrin saturation level might have resulted in overestimates of the prevalence of those who will progress to iron overload in the study population.

In conclusion, elevated serum transferrin saturation has implications for increased mortality risk. The additional mortality associated with elevated serum transferrin saturation would appear to affect many more persons than previously thought. A substantial proportion of the US adult population appears to share this risk.

To read commentaries or to post a response to this article, see the online version at http://www.annfammed/cgi/content/full/2/2/133.

Financial support: This study was funded in part through grant 1D12HP00023-01 from the Health Resources and Services Administration and from a contract from the Delaware Department of Health and Social Services.

Acknowledgments: Special thanks to William Pearson, MHA, and Mark Geesey, MS, for their help with data analysis.

Key words: Hemochromatosis; transferrin; iron overload; cohort studies

Submitted November 21, 2002; submitted, revised, February 7, 2003; accepted February 18, 2003.

\section{References}

1. Fracanzani $A L$, Conte $D$, Taioli $E$, et al. Increased cancer risk in a cohort of 230 patients with hereditary hemochromatosis in comparison to matched control patients with non-iron-related chronic liver disease. Hepatology. 2001;33:647-651.

2. Burke W, Thomson E, Khoury MJ, et al. Hereditary hemochromatosis: gene discovery and its implications for population-based screening. JAMA. 1998;280:172-178.

3. Knekt P, Reunanen A, Takkunen H, Aromaa A, Heliovaara M, Haku linen T. Body iron stores and risk of cancer. Int J Cancer. 1994;56: 379-382.

4. Barton JC, Edwards CQ, Bertoli LF, Shroyer TW, Hudson SL. Iron overload in African Americans. Am J Med. 1995;99:616-623.

5. McCullen MA, Crawford DHG, Hickman PE. Screening for hemochromatosis. Clinica Chimica Acta. 2002;315:169-186.

6. Andrews NC. Iron metabolism: iron deficiency and iron overload. Annu Rev Genomics Hum Genet. 2000;1:75-98.

7. Leggett BA, Halliday JW, Brown NN, Bryant S, Powell LW. Prevalence of haemochromatosis amongst asymptomatic Australians. $\mathrm{Br} J$ Haematol. 1990;74:525-530.

8. Edwards CQ, Griffen LM, Goldgar D, Drummond C, Skolnick MH, Kushner JP. Prevalence of hemochromatosis among 11,065 presumably healthy blood donors. N Engl J Med. 1988;318:1355-1362.

9. Looker AC, Johnson CL. Prevalence of elevated serum transferrin saturation in adults in the United States. Ann Intern Med. 1998;129: 940-945.

10. Baer DM, Simons JL, Staples RL, Rumore GJ, Morton CJ. Hemochromatosis screening in asymptomatic ambulatory men 30 years of age and older. Am J Med. 1995;98:464-468. 
11. McDonnell SM, Hover A, Gloe D, Ou C, Cogswell ME, GrummerStrawn L. Population-based screening for hemochromatosis using phenotypic and DNA testing among employees of health maintenance organizations in Springfield, Missouri. Am J Med. 1999;107:30-37.

12. Beutler E, Felitti VJ, Koziol JA, Ho NJ, Gelbart T. Penetrance of $845 \mathrm{G}$ A (C282Y) HFE hereditary haemochromatosis mutation in the USA. Lancet. 2002;359:211-218.

13. Willis G, Wimperis JZ, Smith KC, Fellows IW, Jennings BA. Haemochromatosis gene C282Y homozygotes in an elderly male population. Lancet. 1999;354:221-222.

14. Niederau C, Fischer R, Sonnenburg A, Stremmel W, Trampisch HJ, Strohmeyer G. Survival and causes of death in cirrhotic and noncirrhotic patients with primary hemochromatosis. N Engl J Med. 1985;313:1256-1262.

15. Fargion S, Mandelli C, Piperno A, et al. Survival and prognostic factors in 212 Italian patients with genetic hemochromatosis. Hepatology. 1992; 15:655-659.

16. Mainous AG III, Gill JM, Pearson WS. Should we screen for hemochromatosis? An examination of downstream effects on morbidity and mortality. Arch Intern Med. 2002;162:1769-1774.

17. Yang Q, McDonnell SM, Khoury MJ, Cono J, Parrish RG. Hemochromatosis-associated mortality in the United States from 1979 to 1992: an analysis of Multiple-Cause Mortality data. Ann Intern Med. 1998; 129:946-953.

18. Witte DL, Crosby WH, Edwards CQ, Fairbanks VF, Mitros FA. Practice guideline development task force of the College of American Pathologists. Hereditary hemochromatosis. Clin Chim Acta. 1996;245:139-200.
19. Edwards CQ, Kushner JP. Screening for hemochromatosis. N Engl J Med. 1993;328:1616-1620.

20. Black WC, Haggstrom DA, Welch HG. All-cause mortality in randomized trials of cancer screening. J Natl Cancer Inst. 2002;94:167-173.

21. Charlson ME, Pompei P, Ales KL, MacKenzie CR. A new method of classifying prognostic comorbidity in longitudinal studies: development and validation. J Chronic Dis. 1987;40:373-383.

22. Shah, BV, Barnwell BG, Bieler GS. SUDAAN User's Manual. Release 7.5. Research Triangle Park, NC: Research Triangle Institute; 1997.

23. McCarthy GM, McCarthy CJ, Kenny D, Crowe J, Eustace S. Hereditary hemochromatosis: a common, often unrecognized, genetic disease. Cleve Clin J Med. 2002;69:224-237.

24. Harris MI, Goldstein DE, Flegal KM, et al. Prevalence of diabetes, impaired fasting glucose, and impaired glucose tolerance in U.S. adults: the Third National Health and Nutrition Examination Survey, 1988-1994. Diabetes Care. 1998;21:518-524.

25. De Beer FC, Hind CRK, Fox KM, Allan RM, Maseri A, Pepys MB. Measurement of serum C-reactive protein concentration in myocardial ischaemia and infarction. Br Heart J. 1982;47:239-243.

26. Ridker PM. Clinical application of C-reactive protein for cardiovascular disease detection and prevention. Circulation. 2003;107:363-369.

27. Sakkinen P, Abbott RD, Curb JD, et al. C-reactive protein and myocardial infarction. J Clin Epidemiol. 2002;55:445-451. 